\title{
Data Fusion for Free-form Surfaces in Reverse Engineering
}

\author{
Peng-xin Liu ${ }^{1, a}$, Hai-li Jia ${ }^{1, b}$, Zhao Wei ${ }^{1, c}$ \\ ${ }^{1}$ Tianjin Key Laboratory of High Speed Cutting and Precision Machining, Tianjin University of \\ Technology and Education, Tianjin, China, 300222

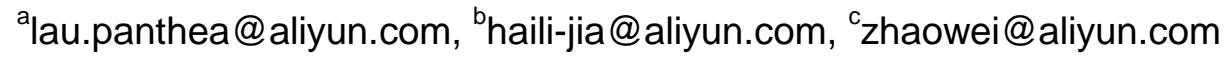

Keywords: Data fusion, Reverse engineering, Multi-sensor integrated measuring system

\begin{abstract}
Reverse engineering is a design process going from a physical or clay model to a digital model. The first step in reverse engineering is part digitization, and its precision and efficiency all will influence the reconstructed surface qualify. In this paper a date fusion method for free-form surfaces using digitized points measured by multiple-sensor integrated measuring system with a touch probe and an optical scanning probe is presented. The optical scanning data will be wrapped in the form of triangulated mesh. Then the special mesh region that needs to be updated is recognized through the detection of the overlapping area between the mesh and the boundary of the newly added data points with high precision captured by a touch probe. After that, the initial triangular mesh is eventually updated to the final output by replacing the recognized region with the newly added data points. The merging data is unified into a precise 3D model in CAD software environment based on the strategy for optimization reconstruction. Experimental results demonstrate the efficiency of the proposed method, which enhances the flexibility of digitizing while maintaining the accuracy of feature surfaces.
\end{abstract}

\section{Introduction}

Reverse engineering can be defined as the process of generating a computer-aided design (CAD) model from an existing part or prototype. While reverse engineering has been used for many years in industry, particularly in the automotive and aerospace sectors, it has shown a marked increase in implementation [1]. This is primarily due to the introduction of rapid part digitization technologies, and the availability of inexpensive computers.

CMM and laser scanning digitization each have a number of contrasting advantages and disadvantages limiting their scope in reverse engineering applications. The combined RE modeling method which couple both data collection methods offer significant flexibility in dictating digitization accuracy, density and process times [2].This flexibility increases the range of reverse engineering applications. This paper mainly focuses on date fusion for free-form surfaces which is the key technology in the combined RE modeling method. The whole process is shown in the following figure.

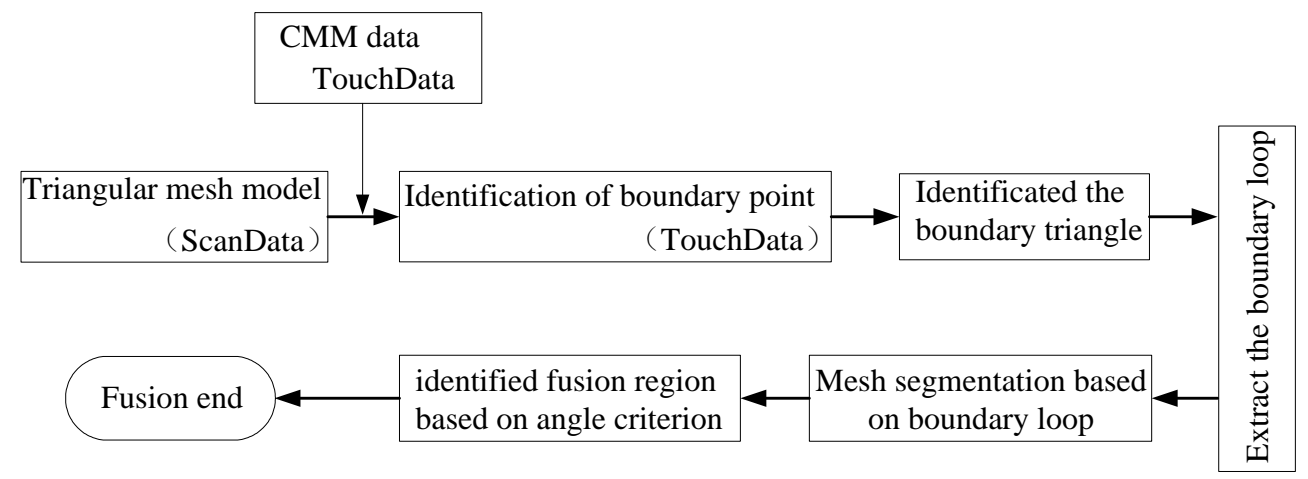

Fig. 1 Overall procedure for data merging

The starting point is the acquisition of a number of clouds of points using optical scanning probe from a part of surface, and these points are exported in the form of a triangulated mesh. It is necessary 
to identify feature surfaces which are measured by a touch probe. The data fusion method which point to the free-form surface is propose in this paper, according to the measured data of CMM to achieve automatic segmentation of optical scanning data and the final data fusion.

It's difficult to determine the segmentation standard of the free surface region, this paper proposed an adaptive fusion method of the CMM data and optical scanning data, at first, identify the boundary points of the CMM measurement data, by means of boundary point to grid model identifies boundary triangle, and then extract boundary ring based on the judgment theory of point in-or-out of polygon, realizes adaptive mesh segmentation based on the boundary ring, at last the recognition of regional integration is realized under the angle criterion.

\section{Identification Boundary Ring}

In order to extract the boundary ring, first need to identify measurement data from CMM boundary point, the boundary point is the measuring point which can express real boundary characteristics of the sample. This paper uses the method of literature extraction of boundary points [3]. The identification of the boundary point from CMM measuring data, boundary points can be determined by the corresponding boundary triangle mesh model. Although some boundary points and mesh model have overlapping area, the grid model is constructed by linear interpolation of the scan data, thus the boundary points do not necessarily fall on the surface of the triangle. Therefore, through the projection method to determine the mesh model and corresponding boundary points on the boundary triangle, the steps are as follows:

(1) For any boundary point $p_{i}$, using Euclidean distance as the standard, search the vertex $v_{j}$ which is nearest to $p_{i}$ in mesh model;

(2) According to the data structure of mesh model to determine the 1- ring neighborhood triangle of $v_{j}$, as shown in Figure 2, all endpoint which belong to the $v_{j}$ in one half of the triangle will be labeled as potential boundary triangle;

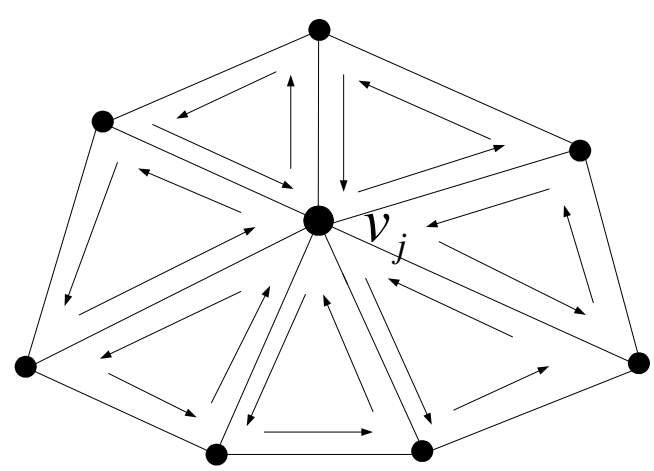

Fig.2 Data structure in 1-ring neighbor

(3) Make $p_{i}$ projected respectively to the 1- ring neighborhood triangle of $v_{j}$, according to area criterion to determine whether the $p_{i}$ project into the triangle, if the $p_{i}$ projection points is in the triangle, the triangle will be the corresponding boundary triangle; if the projection point of $p_{i}$ did not fall on the $v_{j} 1$ - ring neighborhood within the triangle, the triangle $T_{c}$ which has the minimum area error will be selected as the boundary triangle, the barycenter of the $T_{c}$ as the projection point corresponding to $p_{i}$;

(4) Checking all the boundary points, and record the corresponding relationship between projection point and boundary triangle.

The data from CMM are sparse relative to the optical scanning data, thus the corresponding boundary triangle does not form a closed ring, obviously it's not meet the requirements. Therefore, make the line between the projection points as oriented, extract boundary ring based on the judgment method of point in-or-out of polygon theory. 
Based on sorting of the projection point, formation sequence $p_{i}, i=0,1,2 \ldots . n$, the number of projection points is $\mathrm{n}+1$, connected projection points according to the sequence, such as the formation of polygon $P=P_{0}, P_{1}, P_{2}, \ldots, P_{n}$ is shown in Figure 3, the projection point is the vertices of polygon.

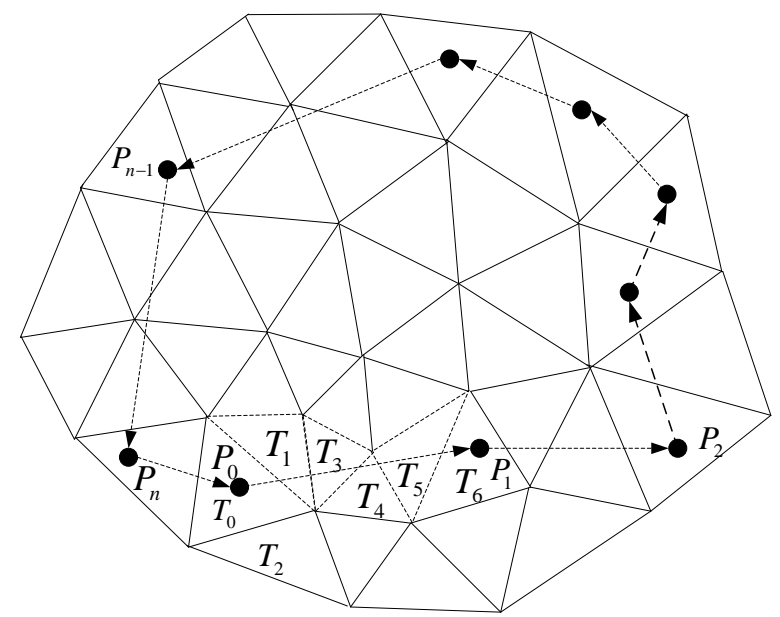

Fig.3 A polygon constructed from project points

If the projection area is flat, polygon $\mathrm{P}$ is planar polygon. For example, projection point $P_{0}$ and $P_{1}$, $P_{0}$ corresponds to the boundary triangles $T_{0}, P_{1}$ corresponding to the border triangle $T_{6}$, edge $P_{0} P_{1}$ through the triangle (dashed line) $\mathrm{T}$ is the boundary triangle which need to be marked. Therefore, a determination condition is given: if two points on either side of the triangle polygon $P$ respectively in the internal and external, then the triangle connected the edge on both sides of the border is boundary triangle.

\section{Adaptive Mesh Segmentation}

Closed boundary ring can be constructed through the extraction operation of the boundary rings; the triangular mesh model is divided into different areas through the boundary rings. In order to divide the model, first choose a triangle which does not belong to any boundary ring as seed triangle, then divided the triangle model by area growth method [5]. There are two categories of triangle in this paper, one is the marked triangle on the boundary ring, and the other is the non boundary ring triangle.

In the process of area growth, the adjacent similar triangle are integrate into the patch with the seed triangle continuously, until the adjacent triangles are all becoming the boundary ring triangle, then to the other side of the boundary ring, the adjacent triangle which is non boundary ring becoming the new seed triangle, repeat the area grouth process. The initial triangular mesh model is divided into $k$ patches at last. $k$ is the index of patch, $k=0,1,2 \ldots m+1, m$ is the number of boundary ring. In the $k$ patch contains the point which need to be replaced by the data from CMM. This process need to be judged by certain criteria, the angle criterion which will be discussed in the following sections of this paper.

\section{Identification Fusion Region based Angle Principle}

The boundary ring is the overlap area between the CMM measurement data and the triangle mesh model; these two areas will coincide at the boundary in measuring process at least. The angle between the CMM data and the surface (waiting fused area) within the boundary ring is less then the angle between the CMM data and the other surface. Fig .4 is an example of the angle, point $A, B$ as the projection point in the boundary ring, $\theta_{1}$ is a angle between the CMM data and the surface within the boundary ring, which is less than the $\theta_{2}$ ( the angle between CMM data and the outside surface of boundary ring). 


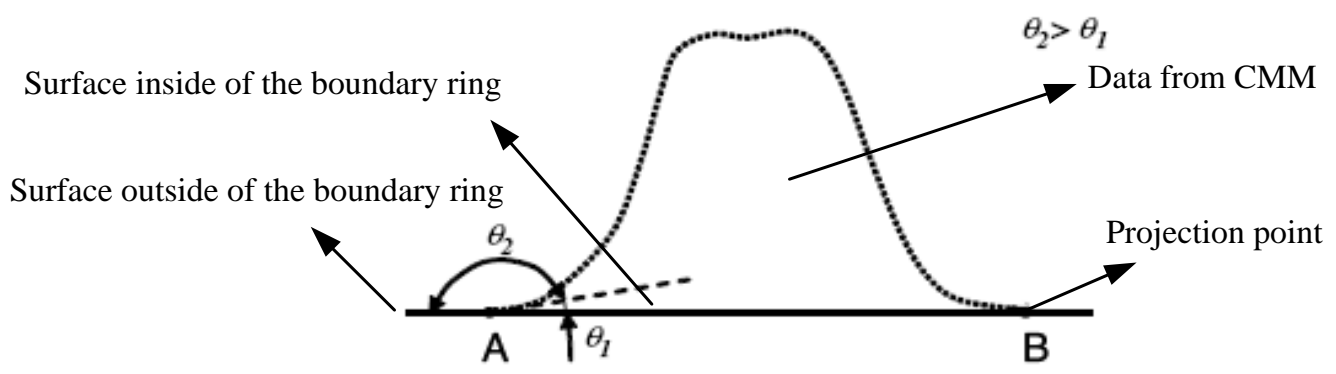

Fig.4 An example of the angle condition

In order to description the algorithm facilitated, gives the following symbols and definitions:

$E_{i}\left(P_{i}, P_{i+1}\right)$ : The line between adjacent projection points $P_{i}, P_{i+1}$ within the boundary ring, also called the boundary edge.

$T_{i}^{k}$ : The triangle which formed with the boundary edge $E_{i}$ and the nearest point to the midpoint of $E_{i}$, where $k$ is the patch index, $\mathrm{i}$ is boundary edge index, as the triangle $T_{i}{ }^{c}$ and $T_{i}{ }^{l}$ in Fig.5 a).

$F_{i}{ }^{m}$ : The triangle is formed by the boundary edge $E_{i}$ and CMM measurement data which is nearest to the midpoint of $E_{i}$ and not a boundary edge point, where $m$ is the number of CMM data sets, $i$ is the boundary edge index.

$T_{i}^{k}$ and $F_{i}{ }^{m}$ are called shared boundary edge triangles

$\theta_{i}{ }^{k}$ : It's the angle between the shared boundary edge triangles $T_{i}{ }^{k}$ and $F_{i}{ }^{m}$, also called boundary angle.

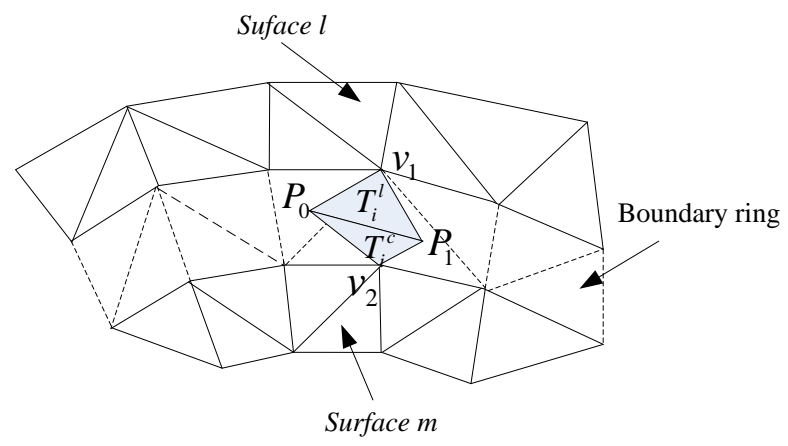

a) shared triangle of boundary edge

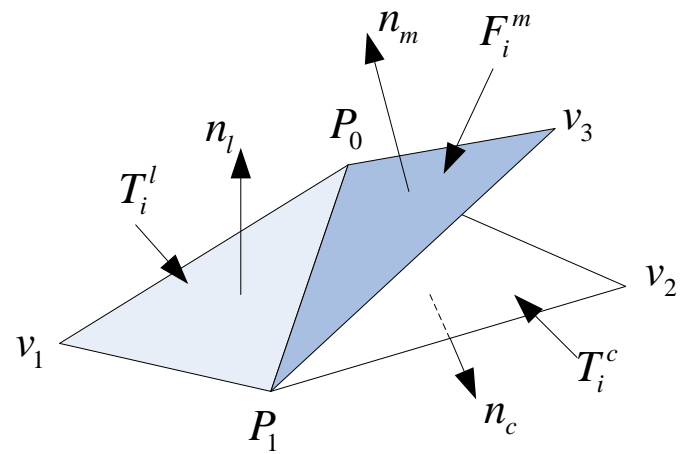

b)relationship between the boundary angle

Fig. 5 Calculation of the boundary angles

$\theta_{i}{ }^{k}$ is calculated by the normal vector of $T_{i}^{k}$ and $F_{i}{ }^{m}$, it's should be consistently in the process. for example, in Fig. 5 b), the formula of normal vector of triangle $T_{i}^{c}, T_{i}^{l}, F_{i}{ }^{m}$ is as followed:

$$
n_{l}=\frac{P_{0} v_{1} \times P_{0} P_{1}}{\left|P_{0} v_{1} \times P_{0} P_{1}\right|}, \quad n_{c}=\frac{P_{0} v_{2} \times P_{0} P_{1}}{\left|P_{0} v_{2} \times P_{0} P_{1}\right|}, \quad n_{m}=\frac{P_{0} P_{1} \times P_{0} v_{3}}{\left|P_{0} P_{1} \times P_{0} v_{3}\right|}
$$

The formula for $\theta_{i}^{k}$ is :

$$
\theta_{i}^{k}=\angle F_{i}^{m} T_{i}^{k}=\cos ^{-1}\left(\frac{n_{F_{i}^{m}} \bullet\left(-n_{T_{i}^{k}}\right)}{\left|n_{F_{i}^{m}}\right|\left|\left(-n_{T_{i}^{k}}\right)\right|}\right)
$$

$\theta_{i}^{k} \leq 0 \leq \pi, \quad \mathrm{i}=0, \ldots, \mathrm{n}, \mathrm{n}$ is the number of boundary edge.

According to the boundary angle $\theta_{i}{ }^{k}$, the following decision angle criterion of regional integration is given:

Suppose $\theta_{\text {mean }}^{k}$ is the average of $\theta_{i}{ }^{k}$, then: 


$$
\theta_{\text {mean }}^{k}=\sum_{i=0}^{n} \theta_{i}^{k} / n
$$

$k$ is patches index, $n$ is the number of boundary edges, has known the patches which the vertex is nearest to the boundary edge are $l$ and $c$, and $\theta_{\text {mean }}^{l}<\theta_{\text {mean }}^{c}$, then $l$ is the patch fusion region.

According to the angle criterion, marked the fusion area, the corresponding data fusion, scanning data will be deleted, replaced by CMM data. Finally, the complete CAD model will be reconstruct based on these fused data under the optimization of reconstruction strategy.

\section{Results and Discussion}

Using the algorithm of this paper, some experiments have done to verify the theory. Figure 6 is a model of data fusion instance.

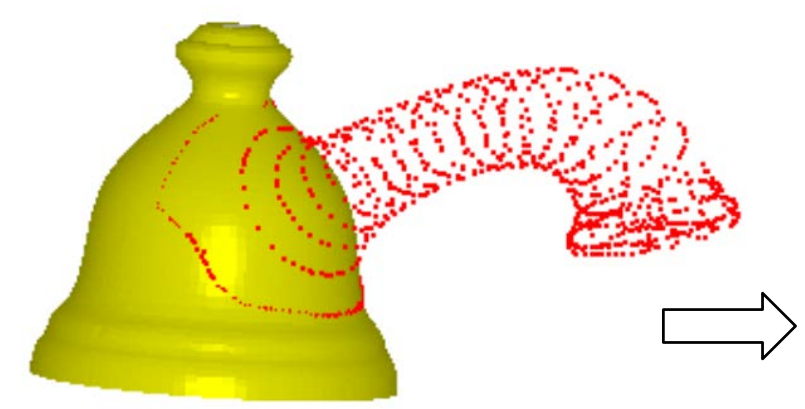

a) The initial mesh model and CMM data

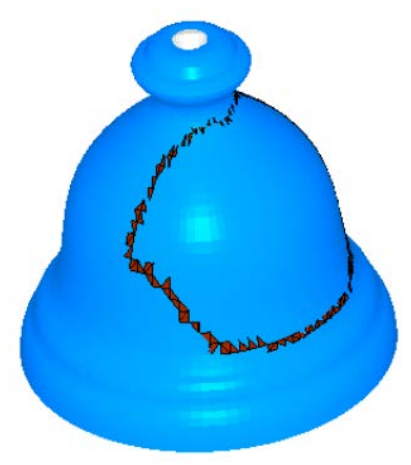

b) Identification of boundary triangles

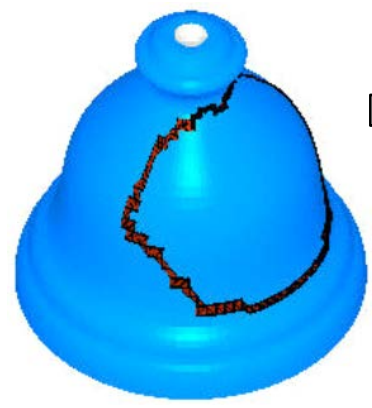

c) Extraction of closed boundary ring

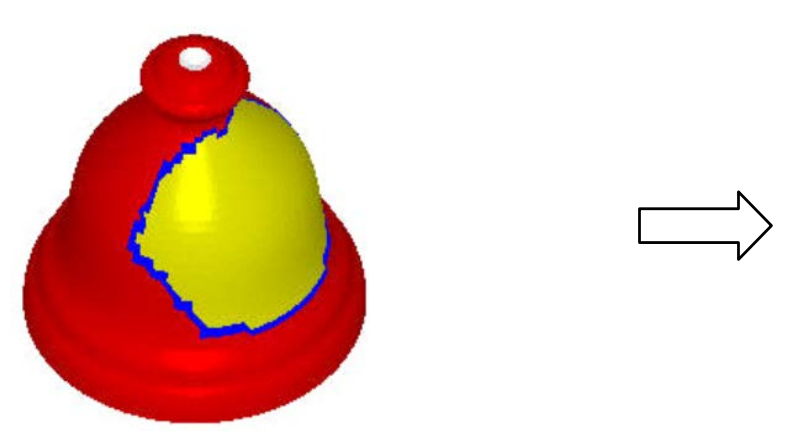

e) Mesh segmentation based on boundary ring

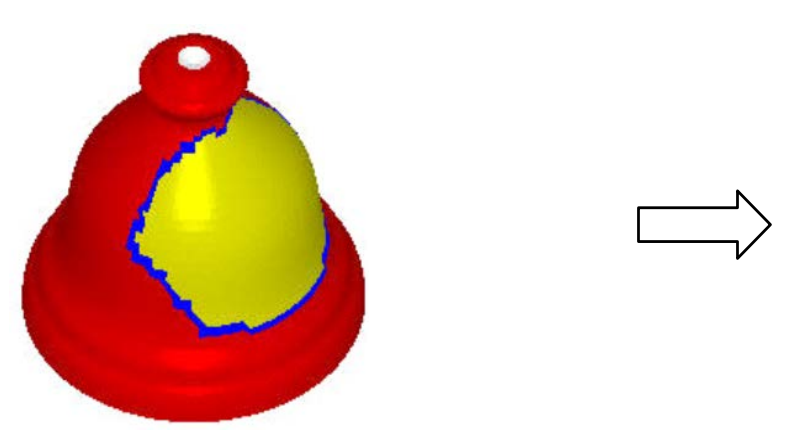

d) The boundary ring amplification

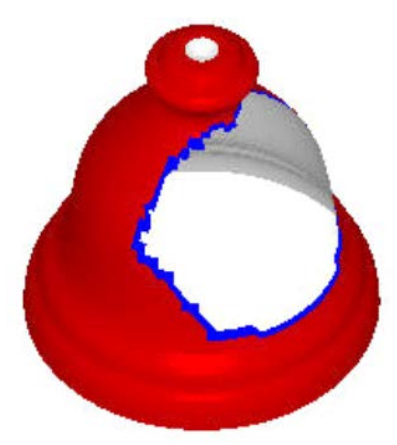

f) Delete the fusion region 


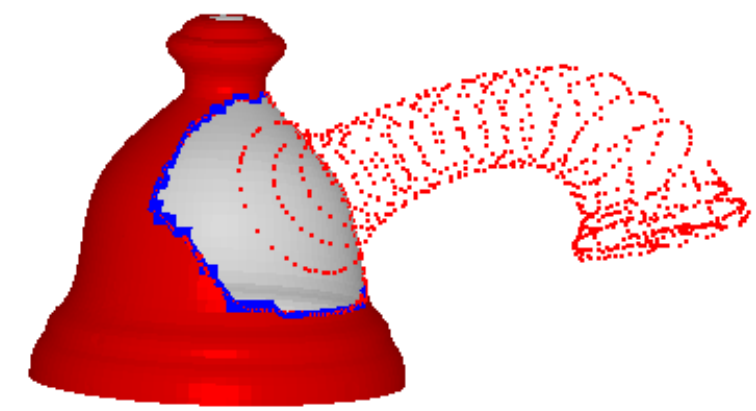

g) The results of data fusion

Fig. 6 An example of a faucet data merging

After the registration, simulation model of CMM data and the corresponding initial mesh model is shown in Fig. 6 a), the boundary triangle recognition results are shown in Fig. 6 b) . According to the boundary extraction algorithm, the discontinuous boundary triangle is closed as a boundary ring, as shown in Fig. 6 c). It can be seen that along the line direction, boundary triangle projection point growth to form a closed ring from the partial enlargement of the boundary ring (Fig. 6 d)). Fig .6 e) is the segmentation results based on grid boundary ring. Integration of regional CMM data corresponding to the identified and deleted based on the angle criterion as Fig . 6 f) shows. Fig. 6 g) is the final result of data fusion.

This method realized the data fusion according to the corresponding data of boundary mesh segmentation; realize the integration of measurement data between CMM data and optical scanning data. In fact, this method can also be used for the fusion between the optical scanning data.

\section{Conclusions}

A method of data fusion is proposed in this paper, the problem of overlapping CMM data and optical scanning data is solved through data fusion. The surface data which can not meet the accuracy requirement of optical scanning are replaced by high precision measurement data from CMM. In addition, the proposed fusion method of free-form surface area data, also can achieve rapid customization of triangular mesh model and deformation.Time for the digital design is shorten, because there is no need to rescan the initial model in this method. It's very meaningful for the deformation design and the free-form surface design.

\section{Acknowledgements}

This work was financially supported by National Natural Science Foundation of China (51505336) and Tianjin University \& Education Foundation (KJ1423)

\section{References}

[1] Ye, X. Z., Liu, H.Z., and Chen, L., et al. Computer Aided Design (2007)

[2] J. Huang, C.H. Menq: IEEE Transactions on Robotics and Automation (2001)

[3] Li Jiang-xiong. Machine Design and Manufacturing Engineering(2000)

[4] He Mei-fang, Zhou Lai-shui, Shen Hui-cun. Journal of Nanjing University of A eronautics \& A stronautics (2005)

[5] H.W.Lin, C.L.Tai, G.J.Wang. Computer-Aided Design(2004) 